\title{
Théologiques
}

\section{Des images (et) de Dieu}

Entre Maître Eckhart et Nishida Kitarō

\section{Marcello Ghilardi}

Volume 20, numéro 1-2, 2012

Les philosophes de l’École de Kyōto et la théologie

URI : https://id.erudit.org/iderudit/1018854ar

DOI : https://doi.org/10.7202/1018854ar

Aller au sommaire du numéro

\section{Éditeur(s)}

Faculté de théologie et de sciences des religions, Université de Montréal

ISSN

1188-7109 (imprimé)

1492-1413 (numérique)

Découvrir la revue

Citer cet article

Ghilardi, M. (2012). Des images (et) de Dieu : entre Maître Eckhart et Nishida Kitarō. Théologiques, 20(1-2), 61-81. https://doi.org/10.7202/1018854ar
Résumé de l'article

Cet article porte sur l'affinité de pensée entre le maître dominicain Johannes Eckhart (1260-1327) et le philosophe japonais Nishida Kitarō (1870-1945) à propos de la dimension de l'image par rapport au questionnement de ce qui se trouve au-delà de la parole, mais qui demeure comme l'objet central de la recherche théologique et philosophique pour les deux penseurs. L'attitude qui soutient une rencontre entre les pensées de Eckhart et Nishida peut réduire les prétentions absolutistes et identitaires qui empêchent un renouvellement de la philosophie et de la théologie. La vérité qui apparaît dans la rencontre de deux penseurs est une vérité qui reste une, mais pluraliste, puisqu'elle sait respecter la différence des identifications religieuses et philosophiques - il ne s'agit pas de les nier en mêlant tout dans un même système indifférencié - et à la fois elle sait garder un fond qui résiste au-delà ou en deçà des différences.
Ce document est protégé par la loi sur le droit d'auteur. L'utilisation des services d’Érudit (y compris la reproduction) est assujettie à sa politique d'utilisation que vous pouvez consulter en ligne.

https://apropos.erudit.org/fr/usagers/politique-dutilisation/ 


\title{
Des images (et) de Dieu
}

\section{Entre Maître Eckhart et Nishida Kitarō}

\author{
Marcello GHILARDi" \\ Philosophie \\ Université de Padoue (Italie)
}

La dimension de l'image et la réflexion portée sur elle constituent une possibilité d'accès conjoint et de liaison entre les différents domaines des pensées et des itinéraires parcourus par Maître Eckhart, d'une part, et Nishida Kitarō, d'autre part. Avec le mot image, on veut faire référence ici au fait qu'on exprime nécessairement "en figure " ce que la recherche philosophique et même la recherche théologique s'efforcent de viser: la Vérité, l'Absolu, Dieu ou, en tout cas, l'Abîme indicible qui est fond de l'être des choses visibles et invisibles. Puisque le langage reste un moyen de viser l'absolu, mais qu'il lui fait écran à la fois, il faut un emploi des mots et du langage dans son ensemble qui soit en mesure d'équilibrer ce rapport difficile entre ce qui peut être nommé et ce qui reste innommable, entre visible et invisible.

De façons différentes, Eckhart et Nishida abordent le thème de l'image — image qui concerne le domaine du visible et à la fois image en tant que dimension rhétorique, c'est-à-dire la parole en tant qu'outil pour signifier l'indicible. Ils forgent tous les deux des termes inédits dans leur langue respective pour rendre compte d'une torsion du langage nécessaire pour communiquer ce qui demeure au-delà de la parole. Nishida n'a pas été un exégète de la pensée de Maître Eckhart ni d'autres philosophes européens.

\footnotetext{
* Marcello Ghilardi est chercheur au Département FISPPA - Philosophie, Sociologie, Pédagogie et Psychologie de l'Université de Padoue (Italie). Ses champs de recherche sont la philosophie interculturelle, l'esthétique, la philosophie japonaise et chinoise contemporaine, le dialogue entre bouddhisme et christianisme. Il fait partie de la commission éditoriale de la revue The Journal of Japanese Philosophy et dirige actuellement l'Institut Confucius de Padoue. Il a récemment publié (2012) Filosofia dell'interculturalità, Brescia, Morcelliana et, avec Enrico Fongaro (2012) Il visibile differente. Sguardo e relazione in Derrida, Milan-Udine, Mimesis.
} 
Dans son travail d'élaboration théorique, il emprunte des intuitions, des idées, des expériences de pensée et il les retravaille pour en tirer des façons nouvelles d'interpréter le monde. C'est ainsi qu'il forge une forme d'intelligibilité inédite. Donc, il faudra poser en parallèle quelques passages de ces auteurs pour faire naître la possibilité d'une nouvelle approche des thèmes de la vision, de l'expression de l'absolu. De plus, l'intérêt de cette confrontation ne s'arrête pas à une simple possibilité de comparaison érudite. Il s'agit plutôt de développer une interaction, d'organiser une sorte d'alternance respiratoire entre deux côtés ou domaines différents, d'appeler une lecture croisée des textes et des cultures, pour tenter d'élaborer une pensée qui résiste dans la tension entre l'un et l'autre, et une théologie qui sache contenir l'écart entre traditions diverses, mais réciproquement fécondes, grâce à plusieurs facteurs. On n'ira donc pas jusqu'à retrouver dans les pages de Nishida des références directes ou explicites aux sermons de Maître Eckhart, mais on cherchera à mettre philosophiquement en tension leurs démarches. À partir de ce dialogue, on visera l'actualité d'une théologie qui ne soit pas un "parler de Dieu", mais plutôt un "parler à Dieu ", en s'ouvrant à une dimension qui rapproche en soi-même le personnel et l'impersonnel, le rapport à l'Autre et la descente dans son for intérieur ou, dit autrement, l'expérience du «pouvoir-autre» (tariki, 他力) et du «pouvoir-du-soi» (jiriki, 自力). Finalement, la rencontre entre une forme de pensée qui provient de l'Europe du Moyen Âge et une forme qui provient de la modernité japonaise peut aider à souligner l'importance soit pour la philosophie, soit pour la théologie — d'envisager la spécificité des cultures en termes de fécondité plutôt que d'identité, ce qui conduirait à se figer dans une fermeture culturelle au lieu d'envisager la richesse de l'altérité et des écarts produits par la pluralité des langues et des traditions (voir Jullien 2008, 219-263).

Si l'expérience de foi et de pensée de Maître Eckhart est enracinée dans la religion et la philosophie de l'Europe, la pensée de Nishida se révèle en elle-même habitée par une tension dynamique. La philosophie de Nishida peut être considérée comme occidentale parce que développée à partir des catégories logiques et épistémologiques européennes; mais les racines de sa pensée se situent dans une expérience préphilosophique qui vient de la langue japonaise (qui n'est pas directement ontologique) et de la nourriture du bouddhisme des écoles zen et Jōdo. Pour cette raison, son expérience de pensée se manifeste comme véritablement interculturelle, puisqu'elle croît et se développe en contenant à l'intérieur d'elle-même la dimension du dialogue et de la tension entre identité et différence. 
On considère donc la dimension du dialogue et de la rencontre comme structurelle et nécessaire, pas seulement comme curiosité ou possibilité d'érudition. Si l'on cherche un dialogue véritable entre des expériences de pensée et de vie, alors il ne doit pas s'agir d'un compromis, mais de la possibilité d'ouvrir et de relancer la dimension de l'bumain dans son ensemble. Il faut que nous soyons conscients du fait que nous pouvons regarder la vérité uniquement du point de vue de ses significations, des notions qu'on utilise pour l'exprimer, des images qui l'indiquent, mais qui la recouvrent à la fois. On ne peut jamais dire la vérité du côté de son événement. En même temps, la vérité ne se donne que par ses figures et par les notions qui l'expriment. Elle fait signe à partir des notions et des figures, mais elle n'est jamais épuisée par elles. La vérité passe dans ses figures, elle ne s'arrête pas en elles, mais en même temps la vérité réside dans ses images. Le lieu dans lequel les images de la vérité se produisent est en même temps le lieu dans lequel ces images tombent et sont relevées.

\section{Image et regard dans la pensée de Maître Eckhart}

Entbildung est un mot très particulier, qu'on retrouve dans les sermons de Eckhart. On peut dire qu'il constitue l'un de ses concepts les plus originaux, même s'il n'est pas cité maintes fois dans ses écrits. D’un point de vue étymologique, ce mot est construit à partir du terme bilde, "dont le champ sémantique est si vaste qu'il couvre et même confond des équivalents latins tel que ratio, forma, phantasma et species" (Wackernagel 1991, 15). Du mot bilde dérivent les notions de Bildung (formation, éducation) et de Umbildung (transformation, métamorphose), mais en premier lieu il passe dans le mot Bild, c'est à dire proprement «image, figure, scène». Bilden et Bildung peuvent composer plusieurs mots dans la langue allemande: Einbildung dit l'imagination (Einbildungskraft est proprement la "faculté imaginative»), Erbildung signifie "construction» dans un sens soit matériel soit métaphorique (voir Wackernagel 1998, 455-456). À la lettre, Entbildung pourrait être traduit avec dés-imagination, libération ou dépouillement de l'image, dé-représentation. Il ne s'agit pas d'une négation ou d'une destruction (Abbau) de l'image: Entbildung désigne pour Eckhart la tentative de décrire le mouvement de retrait de l'être et du rôle des images, pour progresser vers une élévation à la Gottheit, la dimension du «Divin» qui ne peut pas être encadrée dans une nomination définie. L'image ouvre au-delà de soi lorsqu'elle s'évide, lorsqu'elle se rend vide de soi-même. Pour Maître Eckhart, une image est n'importe quoi qui fait 
voile, qui est médiation entre l'âme et Dieu; mais c'est grâce à ce dynamisme entre visible et invisible que Dieu fait irruption (Durchbruch) dans l'âme. On ne doit pas s'étonner alors si sa réflexion à propos de l'image demeure essentiellement éthique et non seulement esthétique. L'image véhicule une polarité sémantique et éthique, qui peut être exprimée uniquement par une formulation linguistique paradoxale:

Il faut donc savoir qu'il n'y a rien d'aussi dissemblable que Dieu et une créature quelconque. Ensuite, deuxièmement, il n'a rien d'aussi semblable que Dieu et une créature quelconque. Enfin, troisièmement, il n'y a rien qui soit à la fois aussi dissemblable et semblable à un autre, que Dieu et une créature quelconque le sont entre eux. (Meister Eckhart 1936a, 110; trad. Brunner 1969, 138; pour ce passage, voir aussi Wackernagel 1998, 459460).

Il faut donc dépasser l'idée d'un dualisme rigide, qui serait incapable de concevoir la possibilité d'une relation authentique entre Créateur et créature. En dépendant ontologiquement de son Créateur, la créature est en effet semblable à lui — d'où la possibilité d'instaurer un autre parallèle: lorsqu'on veut parler de l'art d'un maître, on parle de l'image qu'il a créée, et cette image révèle l'art du maître. Donc l'image n'est pas négative, ne représente pas un obstacle à enlever tout de suite. Au contraire, elle est utile et positive, dans ses limites, en tant qu'elle est une métaphore. Elle transporte au-delà de soi-même vers une dimension transcendante, en ne niant pas l'immanence dont elle est porteuse. Il faut ressentir dans l'œuvre et l'image une transcendance immanente ou à l'inverse une immanence transcendante. L' "au-delà de l'image" se révèle dans l'image (dans la figure, le mot, le son) et, en même temps, cet «au-delà » en dépasse le sens et les possibilités expressives. L'opération indiquée par le verbe entbilden, la capacité de laisser tomber la prétention de dire de manière définitive ce qui dépasse toute faculté de définition, est une condition fondamentale pour purifier, pour libérer l'âme et l'ouvrir à une attitude d'abandon et d'adhésion à la vie. Cela est quasiment paradoxal: il faut abandonner l'idée que l'image, la figure, le mot puissent en soi définir la chose, pour se rendre capables d'approcher librement la chose même; il faut se détacher du monde pour être capable de vivre pleinement dans les situations quotidiennes; il faut ne pas être $d u$ monde pour savoir vivre dans le monde. En effet, le mot Entbildung est lié à ce que Eckhart nomme Gelassenheit; il est à peu près son équivalent dans le domaine de l'imagination et du voir, de la vision intellectuelle. Ce n'est pas un état, mais un procès, une dyna- 
mique de dépouillement des représentations. Le préfixe ent-, couplé à la racine bild, exprime un mouvement dialectique en renouvellement continu. Si bilden signifie "construire, former des images", ent-bilden implique le mouvement contraire, la soustraction des images. L'image est et n'est pas, demeure dans un régime d'ambiguité, est une remise en cours et en discussion continue de notre être au monde, face aux choses du monde. "Voir " ne définit plus l'activité de l'œil ou d'un sujet, mais dit plutôt une transformation ontologique du sujet même. Il faut que l'on se rende vide pour se faire lieu d'accueil, pour être capable de recevoir la pluralité de l'Être. Dans la mesure où l'âme connaît seulement soi-même et est pleine de soi (on pourrait dire qu'elle est hantée par la philopsychia, un amour étroit et égoïste de soi), elle n'arrive pas à connaître Dieu. Il faut qu'elle s'oublie pour voir et pour connaître Dieu, et de cette façon se retrouver aussi ellemême. Voir sans que l'on prétende être un sujet autonome, indépendant et non conditionné signifie remonter aux conditions du voir même. Voir sans être déterminé en tant que sujet contre un objet veut dire voir au-delà de la détermination même. Pour Eckhart, telle est la manière la plus profonde de voir, pour regarder les choses mêmes, pour les regarder en Dieu et pour voir Dieu dans les choses. "Si l'œil avait en soi une couleur quelconque quand il perçoit, il ne percevrait ni la couleur qui est en lui ni celle qu'il n'a pas, mais parce que toute couleur en est absente, il reconnaît toutes les couleurs» (Maître Eckhart 1971, 111).

Cet exemple est $[\ldots]$ associé à l'intelligence dépouillée d'elle-même, c'est-àdire détachée de l'ici et du maintenant, de la ressemblance aux choses temporelles et créées, cependant, même dans la ressemblance à ces dernières, il y a un principe d'unité qui rend toute ressemblance possible, quelle qu'elle soit, et ce principe d'unité transcende la ressemblance elle-même. (Wackernagel 1991, 60)

Ce qui intéresse Eckhart est de retourner au lieu où moi et Dieu sont un, où ils ne sont pas des étants simplement unis; un lieu où ils peuvent vivre d'une manière toujours renouvelée (voir Ueda 1989, 28-29). Le regard dépouillé de toute ingérence subjective voit Dieu dans les créatures, voit le Dieu mortel dans toute expression du monde. Complexio oppositorum: regard divin, regard humain; visage de Dieu, visage des créatures, éternel et impermanent à la fois. Si l'œil doit voir la couleur, il doit être libre de toute couleur. L'œil avec lequel je vois Dieu est le même œil dans lequel Dieu me voit; mon œil et l'œil de Dieu ne font qu'une vision, une connaissance (ce thème sera repris par Nicolas de Cues, dans son œuvre 
De visione Dei: voir De Cues 1986). Dans cette réflexion des regards, l'un dans l'autre, dans cette reconnaissance réciproque, la vision devient tension éthique. Entbildung doit être ethos: non seulement comportement ou habitus, mais aussi domicile, lieu dans lequel l'âme et le regard doivent habiter en faisant abstraction des images figées et périmées, en allant à la recherche d'une "métaphore de l'invisible, c'est-à-dire d'une métaphore dynamique, qui pour s'investir de sens doit se dévêtir d'elle-même [...] En effet, ce n'est que par une approche éthique et même pratique de l'Entbildung que l'abstraction peut devenir une expérience vécue» (Wackernagel 1998, 471-472). Pour cette raison entbilden est un procès qui ne touche pas uniquement à l'image, mais au «moi » qui doit se reconnaître vide. Les puissances de l'âme doivent être dépouillées, car plus elles sont vides et plus elles sont parfaites et peuvent recevoir tout ce que Dieu donne en partage:

Plus elles reçoivent, plus leur joie est grande, plus elles font un avec ce qu'elles reçoivent, en sorte que la puissance supérieure de l'âme est dépouillée de toute chose et n'a rien de commun avec quoi que ce soit, n'accueille rien de moins que Dieu lui-même dans l'abondance et la plénitude de l'être. (Maître Eckhart 1971, 111)

Les choses et leurs images sont créées avec l'âme, donc il faut qu'elles soient dépouillées d'elles-mêmes (ir selbes entbildet), transformées (überbildet) et engendrées par Dieu. Il faut que l'homme même se dépouille (entbildet) de toutes les choses créées (voir Maître Eckhart 1963, 48-70, passim): en vertu d'une dés-appropriation (Entbildung), peut advenir une transformation (Überbildung) de soi-même et de l'image qu'on est par rapport à la source de toute image. De cette façon, nous serons élevés (beben $\bar{u} f$ ) au-dessus de nous-mêmes, tout en restant liés à notre for intérieur. Voilà une dette très importante de Hegel par rapport à la pensée de Maître Eckhart, où l'on peut déjà retrouver in nuce la notion de Aufhebung - même si l'on ne trouve pas le motif dialectique que lui donnera l'idéalisme hégélien (voir Lossky 1973, 37ss.). Selon Wackernagel, le terme úfheben a une fonction équivalente au verbe entbilden, à partir du latin toll re; mais «il serait plus juste de parler d'une Erhebung, terme quasi omniprésent dans la pensée du maître, où il marque le sens et l'aboutissement d'une percée continuelle de l'âme vers Dieu» (Wackernagel 1991, 114-115). Entbildung nomme en tout cas le procès de réduction, d'ablation de l'activité de la conscience, le passage à une forme d'intuition préréflexive, antéprédicative, dans laquelle l'âme (Seele) voit directement son 
propre fond-sans-fond (Urgrund o Abgrund). Et «quant à la relation dynamique entre image originaire et image, pour Eckhart, soit à un niveau intratrinitaire soit entre Dieu et l'âme, dans son être sans image et au-delà de toute image, chaque image est enfin l'image de l'image originaire, privée de toute image, au-delà de toute image» (Ueda 1989, 53).

L'origine véritable des images, le fond à partir duquel toutes les figures se détachent et où elles s'immergent ne se trouve pas dans une transcendance absolue. Dans toute figure limitée, la présence de ce fond immanent et transcendant est protégée. À proprement parler, le fond, le Abgrund n'est pas - il n'appartient pas à la dimension de l'être, il ne peut pas être défini ontologiquement. La question de la vérité des images se renverse dans une attention nécessaire aux images de la vérité: chaque image fournit et permet une expérience particulière de vérité - mais il ne s'agit pas de surmonter l'image en la refoulant, il faut plutôt l'accueillir, passer à travers elle, en reconnaissant le fait que sa valeur est à la fois sa limite. Chaque image est en même temps identique et distincte de ce dont elle est image, en tant qu'elle reçoit et contient le caractère qui fait signe, en son être image. Pour Eckhart, une image n'est pas de soi-même ni par soimême: elle provient plutôt de ce dont elle est image et lui appartient avec tout ce qu'elle est. Le sens ultérieur de l'image est dans l'image, il n'est pas à l'extérieur d'elle. Mais il faut se dépouiller de toute façon de l'image, de manière à ce que cette dernière puisse montrer sa fonction typique; il s'agit de vivre près des images, sans qu'elles soient absolutisées, sans qu'elles soient considérées indépendantes du regard qui les accueille et du fond dont elles tirent leur existence. «Nous savons que Maître Eckhart distingue les images de l'expérience sensible, relative à la théorie de la connaissance, et l'image "mystique", celle qu'on regarde "les yeux fermés" [...], l'image portée et retournée vers la plus profonde intériorité de l'âme: il y a donc image et images»(Wackernagel 1991, 189). En ce sens alors, l'image se métamorphose et, avec elle, le «moi» du sujet s'ouvre et se transforme à son tour, devient un lieu vide de rencontre, se dispose à l'exercice de purification et de dépouillement. Si le vide, le néant — qui coïncide enfin avec Dieu au-delà des images et de la nomination - cesse d'être pensé ontologiquement et s'il est retrouvé dans une dimension éthique, en tant que possibilité de tout exercice, de toute ascèse, de toute pratique, alors les dualismes s'absorbent et on retrouve aussi un contact direct avec l'expérience pure de la réalité.

Autrement dit, chaque image comporte une erreur, elle ne possède pas la vérité, mais elle permet le passage, le mouvement, le dynamisme de la 
vérité qui se donne justement dans ses figures, mais qui ne s'arrête jamais dans nulle figure. "L'expérience de la vérité vit de ce donner signe, jamais en dehors du signe " (Sini 1985, 174, nous traduisons): toute signification reste dans la chaîne infinie des interprétations, mais l'événement de la signification, le Néant qui persiste au dehors de l'Être et qui permet à l'être de se donner, sont au-delà des interprétations et des images, des figures et des notions qu'on continue à forger pour en parler. Il n'y a pas, en conséquence, une solution théorétique, mais une réponse éthique. On nous demande d'habiter le seuil de l'image, plutôt que de tenter uniquement d'enfoncer son statut ontologique. Toute vérité qu'on peut dire n'est pas la vérité, parce qu'elle renvoie à l'horizon dont elle surgit, dont elle se détache. Toute vérité humaine prend son sens du fait qu'elle passe, se donne et se retire, est à prendre et puis à rendre à son mouvement. Connaître n'est pas alors begreifen, une préhension conceptuelle et analytique, mais plutôt une expérience soit du corps soit de l'esprit - où le dualisme corps/esprit se dissout ou, mieux, ne surgit pas. Et le mot «Dieu» ou «Divinité ( Gottheit) ne sera proféré d'une manière correcte que lorsque nous serons capables de l'engendrer en nous-mêmes; quand, à partir du silence, du détachement le plus profond, le Verbum sera engendré dans l'âme - quand nous serons cette Parole.

\section{Image et lieu dans la pensée de Nishida Kitarō}

La pensée de Maître Eckhart a suscité un grand intérêt chez plusieurs penseurs japonais, notamment les représentants de l'école de Kyōto (Kyōto gakuha) - in primis, chez Nishida Kitarō, le premier à mettre en forme une philosophie japonaise capable de conjuguer catégories occidentales et expérience vécue de la tradition nippone. Même si Nishida n'a pas approfondi, dans des essais ou des études philologiques et spécialisées, l'œuvre de Eckhart, on peut dire que la figure du maître dominicain est présente dans beaucoup de pages du philosophe japonais ${ }^{1}$, à partir de son premier ouvrage, Zen no kenkyū (voir Nishida 1911). Tout au long de son projet philosophique, en effet, Nishida veut déconstruire les dualismes bien enracinés dans la pensée occidentale: sujet et prédicat, sujet et objet, matière et forme, universel et particulier (voir Ghilardi 2005; Ghilardi 2009, 132187; Heisig 2000; Heisig 2001, 55-112; Tremblay 2000, 19-24; Tremblay

1. Voir NKZ $1: 185,186,192,366$; NKZ 6: 159, 181, 182; NKZ 14:311-327; NKZ $17:$ M. 38, M. 40, 1.1 
2007b; Ueda 1977). Il veut reconduire ces dualismes à un «lieu» originaire, qui soit logique, ontologique, épistémologique, pour les circonscrire et les dissoudre dans ce fond, dans cet horizon ineffable. C'est en particulier pendant les années 1920 que Nishida utilise plusieurs fois les métaphores de l'image et du miroir (voir Nishida 1923; 1926). Dans son essai «Basho» (場所), qui, en 1926 représente une sorte de tournant capital dans sa démarche, Nishida utilise la métaphore du miroir, assez typique aussi dans la littérature du bouddhisme zen. Le "lieu du néant absolu» (zettai mu no basho, 絶対無の場所) est visé à la manière d'un miroir qui se reflète en lui-même. En ce sens-là, tout ce qui existe, matériel ou spirituel, visible ou invisible, devient une image ou, à proprement parler, une «image reflétée » ou «image-ombre» (eizo , 影像; kage, 影). Il s'agit alors d'une sorte de jeu de miroirs qui se résout dès que le miroir ultime se dissout en tant qu'objet de pensée, en laissant le singulier se refléter en tant qu'expression du néant absolu.

Quand le lieu [basho] même devient néant [...] tout devient image reflétée $[e i z \bar{o}]$. Lorsque le substrat, sujet qui ne devient prédicat, devient néant, du point de vue du jugement on ne peut que parler d'une image reflétée [eizō] sans aucune substance. Une substance ne peut être retrouvée nulle part, il y a seulement quelque chose qui, en tant que néant, reflète en soi-même sa propre image [eizō]. (Nishida 1926, 445, nous traduisons)

Est intéressante aussi l'attention que Nishida porte aux métaphores du regard, de l'œil et de la couleur, une métaphore chère à la tradition de la mystique européenne, de Maître Eckhart à Nicolas de Cues. Quelques pages auparavant, Nishida avait écrit:

Est-ce qu'on peut concevoir alors une couleur particulière en tant qu'acte de la couleur en soi? Une chose telle que la couleur en soi n'est pas encore quelque chose qui agit, ne comprend pas en soi-même des relations temporelles. Tout simplement, l'universel comprend en soi le particulier et le particulier est dans l'universel. Comme on dit que ce qui a une forme [katachi, 形] est image [kage] de ce qui n'a pas de forme, il s'agit peut-être d'une relation dont les formes infinies se constituent à l'intérieur du même espace sans forme [...] Le lieu véritable devient quelque chose qui reflète en soi-même son image, un miroir qui s'illumine soi-même. (Nishida 1926, 428 , nous traduisons)

$\mathrm{Ce}$ «lieu» est le sans-forme qui réfléchit en soi toutes les formes et qui permet aux formes d'être. En ce sens, on peut penser à résorber aussi l'opposition autrement irréductible entre nécessité et possibilité: d'une 
part, il est nécessaire que ce qui est soit dans un lieu; d'autre part, ce lieu laisse être les choses, en tant que ses déterminations, en tant que négations déterminées. En se référant au lieu qui se reflète dans ses images, Nishida renvoie à une célèbre figure de la tradition bouddhique, la lumière de la lune qui se reflète sur l'eau et rend sa surface claire et lumineuse, sans perdre sa clarté originaire.

Le lieu véritable devient comme ce qui reflète en soi-même son ombre, comme un miroir qui s'illumine soi-même [...] Refléter signifie laisser les formes [katachi] des choses se constituer telles qu'elles sont, sans les déformer; cela signifie les accueillir telles qu'elles sont. (Nishida 1926, 429, nous traduisons)

Tradition bouddhique, référence implicite aux écrits de la mystique rhénane et platonisme convergent dans la pensée de Nishida, alors qu'il essaie de trouver un fond commun soit pour l'être des objets soit pour la volonté des sujets:

La volonté est vue dans le lieu du véritable néant, mais la volonté est encore simplement un aspect de l'acte réfléchi dans le miroir du néant. Nous voyons la volonté dans la mesure où nous voyons le lieu de l'être déterminé. Dans le lieu du véritable néant doit être niée la volonté même, l'acte devient quelque chose de réfléchi et en même temps la volonté aussi devient quelque chose de réfléchi. Ce qui se meut, ce qui agit doit être en général image [kage] de l'éternel. (Nishida 1926, 463, nous traduisons)

On sait déjà, à partir de son premier ouvrage Étude sur le bien (1911) que Nishida considère l'expérience comme une dimension unitaire, dans laquelle le dualisme typiquement occidental entre corps et esprit tombe ou, mieux, n'apparaît pas jusqu'au moment de la réflexion (hansei, 反省) qui se détache de l'intuition (chokkan, 直観), c'est-à-dire du moment du flux où «je» et «tu», sujet et objet ne font qu'un, et où la réalité même est unifiée (tōitsu, 統一). Connaître est toujours comprendre avec tout le corps (taitoku suru, 体得する), ce n'est pas simplement une préhension conceptuelle. «Expérience» (keiken, 経験) renvoie à un procès, à un écoulement, à la distension d'un tissu unitaire qui est toujours en train de se tisser (kei, 経). Mais en japonais on peut utiliser aussi le mot taiken (体験), où tai implique le corps physique, l'être constitutif, par rapport au mouvement libre de la respiration, du souffle, de l'énergie vitale ( $k i$, 気) qui ne se localise pas d'une manière définitive, mais circule librement. Il n'y a pas de fracture entre deux modalités de l'être ou deux structures ontologiques — visible et invisible, sensible et intelligible - il y a continuité, circulation. On pourra trouver des correspondances fécondes, par exemple, avec la 
pensée arrivée à sa maturité de Merleau-Ponty, qui a essayé de surmonter le dualisme corps/esprit, visible/invisible avec sa notion de chair et une idée dynamique et innovatrice d'ontologie, à partir de sa démarche phénoménologique (voir Merleau-Ponty 1964a, 71ss.; Merleau-Ponty 1964b). L'esthétique se trouve donc à la base de toute entreprise de pensée, pas seulement comme théorie de la perception et comme philosophie de l'art, mais plutôt en tant que fond à partir duquel on commence à penser: le corps est une dimension impossible à cerner, à circonscrire, il renvoie au monde et s'unit à lui. Corps et monde forment un couple indissociable, l'un renvoyant à l'autre et existant justement à partir de l'autre. La sensation, notamment la perspective inaugurée par la vision (et parfois le toucher: voir Nishida 1923, 288, 324, 327), conduit Nishida à considérer la leçon hégélienne selon laquelle il n’y a jamais de perception immédiate, simplement passive, de réception des données extérieures auxquelles on peut appliquer a posteriori des catégories interprétatives; la perception est toujours médiation. Un passage de l'œuvre Art et morale est très clair à ce propos, en indiquant dans le voir une métaphore efficace pour faire allusion à toute forme d'intuition et d'action:

Je voudrais essayer de dire que même inférer est un type de vision [...] L'espace intelligible où agit la pensée est encore plus vaste que la perception du voir et du toucher; néanmoins il y a quelque chose d'essentiellement identique. Même si l'espace de la perception du voir est plus vaste, si l'on compare avec l'espace de la perception du toucher, il s'agit du même espace. (Nishida 1923, 439)

Au-delà des médiations des différents types de perception, au-delà des figures qui nous permettent d'entrer en contact avec le monde qui nous entoure, il y a une dimension qui est indéfinissable - et dont on ne devrait pas dire qu'elle est "au-delà » de quoi que ce soit, parce qu'il ne s'agit pas d'une "chose» ou d'un étant, quel qu'il soit. La perspective de Nishida, entre les essais qui composent Art et morale et le texte capital intitulé «Le lieu ", assigne une grande importance au sens de la vue (shikaku, 視覚) et en général au voir (miru, 見る), en soi ou en tant que métaphore de la compréhension $^{2}$.

2. Plusieurs termes de la tradition bouddhique japonaise font allusion au «voir " pour dire la faculté de comprendre la nature foncière de la réalité: $k a k u$ (覚, comprendre, être conscient, éveil) montre dans la partie inférieure du caractère le sinogramme du verbe «voir» (見); kenshō (顕正, éveil, illumination) exprime le «voir correctement » ou «directement». En outre, dans chokkan (直観, intuition) le deuxième caractère chinois présente le radical du verbe "voir» (見, voir Nishida 1923, 267-268). 
À partir de la pluralité des images et des figures de la vérité, on nous demande de les accepter tout en sachant aussi nous détacher d'elles. On verra alors la réalisation de la grande analogie érigée dans le Avatamsaka sūtra, le "Sūtra de l'ornementation fleurie» (Huayanjing en chinois, Kegonkyo, 華巌経, en japonais) à la base de l'école bouddhiste Kegon, dont Nishida apprécia les textes et la tradition de pensée. Ce sūtra traite de la vacuité de tous les phénomènes et de la nature inconcevable de la réalité transcendant les notions d'un et de multiple. On imagine la réalité à la manière d'un filet infini, accroché de telle sorte qu'il s'étende à l'infini dans toutes les directions. Un joyau étincelant est suspendu à chaque nœud du filet et, sur la surface brillante de chaque joyau, se reflètent tous les autres joyaux de la toile, infinis en nombre. Chacun des joyaux réfléchis dans ce joyau singulier reflète tous les autres de telle sorte que le processus de réflexion est infini: on parle à ce propos de la "non-obstruction» entre joyau et joyau, entre image et image, entre phénomène et phénomène (jiji$m u g e$, 事事無碍). Chaque élément est en même temps forme et contenu, un et multiple, condition et conditionné. La vérité de la totalité se reflète et dépasse, perce tous les phénomènes; elle ne consiste pas en un phénomène particulier, elle ne se fige pas dans une figure, un mot, une phrase, mais circule dans toutes ses images et ses figures. Il n'y a pas de vérité sans ses images, mais la vérité n'est pas limitée à une de ces images. Par contre, dans le plus petit des gestes ou des fragments l'Univers entier est contenu et exprimé. Là, c'est Dieu même qui vit, comme Nishida l'avait souligné dans les pages de son Étude sur le bien, à propos de l'intuition artistique et de la capacité de l'artiste à ressentir l'unité profonde de la réalité. Dans cet état, Dieu est le monde et le monde est Dieu (voir Nishida 1911).

\section{Eckhart et Nishida: entre visible et invisible}

Ni Nishida ni Eckhart ne veulent proposer une dimension du Divin en tant que totalement immanent ou une forme de panthéisme. Pour les deux penseurs, le point capital réside dans la capacité de renoncer à son $e g o$, à l'attachement au moi. Atteindre le soi véritable (shin no jiko, 真の自己) ou le soi auto-éveillé (jikakuteki jiko, 自覚的自己) signifie le comprendre en tant que «centre de perspectivité du monde» (Tremblay 2003, 17), le rendre en tant que jaillissement continu du monde et de la vie. Dans son sermon Modicum et iam non videbitis me, Eckhart, en soulignant le rôle de la raison en tant que puissance de l'âme détachée du temps et de l'espace, dit qu'elle est une "image » qui opère en soi-même grâce à la nais- 
sance éternelle de Dieu, avec son Fils. C'est en effet la doctrine traditionnelle de la Trinité qui permet à Eckhart de soutenir l'idée de theiosis sans tomber dans le panthéisme (voir Vannini, 2011, 20-21).

Chaque chose est image, est événement du lieu qui excède toujours toutes les figures et toutes les représentations qui essaient de le dire. La dimension du Divin, la Gottheit, coïncide pour Eckhart avec un Néant; pour Nishida le «Lieu» est «lieu du néant absolu» (zettai mu no basho) puisque rien de ce lieu, de ce prédicat qui ne devient jamais sujet, horizon englobant toute chose, ne peut être réduit à une donnée, à un concept, à une notion quelconque. Cette Gottheit, ce «lieu» n'est rien d'autre Nicolas de Cues dira non aliud - que tout ce qui se passe en lui, néanmoins n'est jamais seulement la totalité de tout ce qui se passe. Le langage qui cherche à l'exprimer arrive toujours après coup; il en est une occasion bien sûr, il est l'une de ses possibilités, de ses occasions, et en tant qu'occasion le langage aussi peut fournir une trace véritable du fond d'où il jaillit. Mais toute vérité que l'homme peut dire n'est pas la vérité unique, parce qu'il s'agit d'une vérité qui renvoie au vide d'où cette vérité surgit. Cette vérité humaine reste toujours et justement en dette par rapport à ce fond. Donc, dire, entendre ce «lieu » ne signifie pas chercher à l'inscrire dans un discours, trouver une réponse théorétique, mais plutôt correspondre à son silence, c'est-à-dire le mettre (et se mettre) en exercice, sans la prétention d'en cerner les significations par des paroles. Du «Lieu », du Dieu, on peut avoir une approche éthique plutôt que théorétique, avec une forme de praxis qui ne fait pas des termes du langage des substances, mais qui sait accueillir les occasions infinies de vie disponibles dans ce fond. Se disposer à cette forme d'exercice veut dire s'ouvrir au vivre, avec la conscience de la réciprocité féconde entre pratique et théorie, intuition et réflexion, action et contemplation.

On pourrait décrire ce mouvement comme une dialectique sans synthèse ou un procès d'éloignements et de rapprochements continus, assez similaire à la dynamique exposée par Jacynthe Tremblay à propos des pensées de Nishida et de Karl Rahner:

L'absolu de Nishida, qui doit se nier absolument lui-même pour se tourner vers le relatif, correspond au Dieu de Rahner qui, par son autocommunication absolue, se révèle dans le fini et comme fini. En revanche, le relatif de Nishida doit se nier absolument lui-même afin de faire face à l'absolu, tout comme l'humain et le monde se remettent totalement au mystère absolu chez Rahner, dans un mouvement où l'autotranscendance de la matière et 
de l'esprit rencontre pleinement le mouvement d'autocommunication de Dieu. (Tremblay 2000, 145)

L'expérience de pensée d'un philosophe japonais comme Nishida peut nous aider à retrouver et à souligner, à partir d'un dehors de l'Occident — c'est-à-dire le langage japonais et la pratique du zen - certains traits fondamentaux de la pensée théologique chrétienne et de son caractère structurellement dialogique, capables d'offrir de nouvelles perspectives et de stimuler à la recherche des penseurs si lointains (voir Ueda 1965, Suzuki 1957). À partir de la démarche de Nishida, on peut considérer à nouveau la dialectique eckhartienne entre la tendance à l'unité et le détachement (Abgeschiendenheit), d'une part, et la capacité de se tourner vers les autres, vers la communauté et la pluralité, d'autre part.

À l'inverse, passer à travers des textes fondamentaux de la tradition chrétienne aide à pénétrer dans la pensée nishidienne, qui reste autrement obscure si l'on ne comprend pas ses liens avec la spéculation d'un Plotin, d'un Pseudo-Denys ou d'un Maître Eckhart. L'obscurité aveuglante du Pseudo-Denys, l'Abgrund d'Eckhart, la caligo du Cusain ou de l'auteur anonyme du texte anglo-saxon Le Nuage de l'inconnaissance sont assumés dans les pages de Nishida, le plus souvent sans référence directe au contexte théiste dont elles proviennent. De façon similaire, l'expérience du zen est pour Nishida une base pour un intérêt spéculatif qui n'existe pas dans la tradition des maîtres bouddhistes japonais. Si l'on cherche chez Nishida une pensée typiquement bouddhique, on ne la trouvera pas; et si l'on croit voir en lui un épigone de l'ontothéologie ou de la mystique spéculative, on ne pourra pas comprendre son originalité. Le risque de sa pensée porte sur la tentative de produire une rencontre et une fécondation mutuelle de langages, catégories et formes d'expériences qui appartiennent à des contextes différents. Son pari philosophique est la possibilité d'indiquer le fond commun à toute expérience, à toute image ou phénomène - comme un doigt qui indique la lune: le doigt a son rôle à jouer en montrant la clarté lunaire et en ce sens il lui appartient, mais il ne faut pas le confondre ou le superposer à cette clarté. Il est une occasion pour tourner le regard, pour percevoir la circulation de la lumière à travers les corps qu'elle entoure. Dans ce doigt, dans cette figure en relation avec la lumière, on trouve toujours la lumière même, le néant absolu dont elle surgit, Dieu qui fait de lui-même signe et qui, en se manifestant, se retire à nouveau, s'absente, demeure insaisissable. 


\section{Du dialogue entre visions du monde}

Ce qui échappe facilement à la possibilité de construire une rencontre et un véritable dialogue entre langages, expériences, cultures et visions du monde différentes est la fécondité de la diversité, voire l'enrichissement qui vient de la confrontation avec l'autre. On est toujours prêts à penser le dialogue comme une nécessité, comme un pari ou un défi, mais on a l'impression que tout le monde serait plus à l'aise si l'on pouvait s'entendre avec une langue, dans une même Weltanschauung, dans un seul ordre de pensée. On ne peut pas oublier, en effet, que si je traduis par «Dieu» ou par «esprit» le mot japonais kami (神), « je mobilise de part et d'autre des pans latents de signification qui ne se recoupent que partiellement» (Jullien 2008, 202). Même si l'on cherche à donner de l'espace à l'écoute de l'autre, en visant à l'universalité sans la présupposer comme donnée d'emblée, on risque toutefois de tomber dans le piège d'une conception monoculturelle et monolinguistique du monde et de la vie. Le langage n'est pas un véhicule neutre et le monolinguisme conduit toujours à des formes plus ou moins évidentes de colonialisme, même si c'était seulement un colonialisme des idées ou des notions (voir Derrida 1996). "Il n’y a pas de dialogue œcuménique qui se passe dans une langue seulement. Penser que par une seule langue l'expérience humaine devienne compréhensible est peut-être le dernier, inconscient reliquat de l'impérialisme culturel» (Panikkar 2009, 180).

Il n'y a pas de mots qui possèdent en soi une valeur ou une nature immédiatement transculturelle. On peut utiliser plutôt des mots, des notions et des catégories en en élaborant le sens, au fur et à mesure, dans une perspective interculturelle. Cela signifie considérer les mots non seulement en tant que signes, mais surtout en tant que symboles, expériences de circulation, de traversée, de passage et de transformation. Personne ne peut avoir le monopole sur un symbole, parce que tous les symboles requièrent une implication personnelle et nouvelle, au-delà des définitions données. À la différence des concepts, les symboles se produisent toujours à nouveau dans la transformation qu'ils suscitent dans les gens qui décident de les rencontrer. Les symboles consistent dans cette circulation et dans cette métamorphose qui se passe dans l'histoire, mais qui emporte aussi au-delà de l'histoire. La pluralité des circulations et des approches que les symboles encouragent conduit à une attitude de clarification réciproque et de mise en évidence des contextes à partir desquels chaque individu commence son mouvement de recherche et de connaissance. En découvrant d'autres sym- 
boles, d'autres langages, on sort de son propre «mythe» (voir Panikkar 2008), on assume une distance critique consciente à l'égard de l'horizon, qui n'est jamais thématisé, mais qui contient et détermine l'ensemble des autoreprésentations que nous avons de nous-mêmes et du monde, pour pouvoir retourner à nous et à ce monde, après que nous ayons renouvelé notre regard.

Tout cela signifie aussi qu'un caractère essentiel de la vérité consiste dans sa disposition à la coopération plurielle des langues et des cultures. Pour être et pour avoir un sens humain, la vérité ne peut pas être coupée de la relation aux personnes, dans leurs identités variables et dynamiques, relationnelles à leur tour. Il n'y a pas de sujet, de langue, de discipline ou d'institution qui puisse posséder la vérité en tant qu'objet; donc, celle-ci ne peut pas être détachée de la relation vive avec les images, les rencontres, les vies qui font qu'elle se présente. Pour cette raison, la rencontre avec une pensée autre devient une occasion majeure de redécouverte de ce qui pourrait autrement passer pour une pensée connue, prévisible et par rapport à laquelle on devient quasiment aveugle.

C'est alors la traduction, patient exercice de confrontation, de dialogue, de sortie de ses catégories linguistiques, expérience d'enchevêtrement et d'échec aussi, qui peut représenter aujourd'hui l'un des modèles les plus utiles et instructifs pour une éthique à construire, soit pour les échanges quotidiens entre êtres humains, soit pour une nouvelle démarche de la pensée théologique du contemporain:

Car la traduction est la mise en œuvre exemplaire de l'opérativité propre au dialogue: elle oblige en effet à réélaborer au sein même de sa propre langue, donc à reconsidérer ses implicites, pour rendre celle-ci disponible à l'éventualité d'un sens autre, ou du moins dans d'autres ramifications [...] La traduction, à mes yeux, est la seule éthique possible du monde «global» à venir. Car si la communication se fait dans la langue d'un des partenaires, ou sans que l'autre langue soit en même temps entendue, la rencontre de ce seul fait est biaisée, s'opérant sur le terrain — et donc dans le jeu des implicites culturels — de l'un des deux. (Jullien 2008, 248)

Il faudra une éthique de l'écoute, de la rencontre, dans un monde pluriel dans lequel les matériaux, utilisés jusqu'à ce moment-là pour ériger des barrières religieuses et politiques, seront employés pour construire des ponts et des passages, pour ouvrir des frontières. Traduire ne veut pas dire simplement passer d'une langue à une autre, mais repenser et coordonner chaque fois un système entier de concepts, d'expériences du monde, de 
relations avec sa propre identité, proposer une généalogie des termes et des notions qu'on emploie souvent distraitement. Traduire permet donc à la dimension inépuisable de la vérité d'émerger, de donner sa richesse infinie.

\section{Conclusion}

Il faut reconnaître que le pluralisme, comme le dit Raimon Panikkar,

[...] n'est pas la justification d'une pluralité d'opinions, mais la perception que le réel est plus que la somme de toutes les opinions possibles [...] En d'autres termes, aucun groupe, aucune vérité, aucune société, idéologie ou religion peut avancer une prétention totale sur l'Homme, parce que l'Homme demeure toujours insaisissable, incomplet, non fini, infini - encore en formation, en marche, itinérant. (Panikkar 2009, 49-50, nous traduisons; voir aussi Panikkar 2002)

Le pluralisme n'est pas une méthode, une idéologie ou un système, mais une attitude essentielle de l'humain qui est conscient de l'irréductibilité des systèmes divers qui servent à rendre compréhensible la réalité, et du fait qu'il n'est pas nécessaire de réduire cette réalité à un seul centre d'intelligibilité (voir Panikkar 2009, 57; Ghilardi 2012, 140-141). L'attitude qu'une rencontre entre les expériences de pensée telles que celles d'Eckhart et de Nishida nous offre peut vraisemblablement réduire les prétentions absolutistes et identitaires qui empêchent tout effort de transformation interculturelle de la philosophie et de la religion. La vérité qui apparaît à l'occasion de la rencontre de deux côtés - le Moyen Âge allemand et la modernité japonaise - est une vérité qui reste une, mais qui sait se rapprocher du contexte. Elle n'est donc pas plurielle, mais pluraliste, puisqu'elle sait respecter la différence des identifications religieuses et philosophiques - il ne s'agit pas de les nier en mêlant tout dans un même système indifférencié - et, à la fois, elle sait garder un fond qui résiste au-delà ou en deçà des différences. Le croisement qu'on a proposé ici essaie de montrer l'importance d'une possibilité autre, par rapport au paradigme métaphysique dominant, dans la relation entre pensée, être et Dieu. Soit pour Eckhart, soit pour Nishida, la pensée cesse de dominer l'être, et se découvre accueillie par un Fond, un Néant, un Lieu qui n'appartient nécessairement ni à la tradition bouddhique ni à la tradition chrétienne, mais qui entoure toutes les traditions et demeure incontournable. En même temps, il reste que chez Eckhart, le caractère mystique de la voie fait accéder à la dimension trinitaire, qui demeure intrinsèque à toute réalité. La 
voie pour parvenir à l'expérience intime du réel coïncide avec son sens ultime - la voie est la vérité dans toutes ses figures, odos, aletheia et zoé se rejoignent (voir Jean 14, 1-6). Pour Nishida, par contre, le procès de la compréhension ou de l'éveil à soi, en se développant en affirmation, négation et négation de la négation, ne s'appuie pas sur une pensée ontologique ou sur une relation trinitaire, à la façon de la mystique chrétienne. Le lieu en tant que néant absolu absorbe toute image et aussi l'image du néant même.

L'être humain et les images qui le décrivent, et qu'il contribue à élaborer pour construire des significations nouvelles grâce auxquelles il peut habiter le monde, sont les fragments d'un entier qui le surpasse, qui n'est jamais susceptible d'être compris par un angle particulier. Mais chaque fragment est essentiel à l'infini qui l'englobe, et il faut en prendre soin, même s'il semble inutile - comme les morceaux de pain qu'il faut garder : «Ramassez les morceaux qui restent, pour que rien ne soit perdu» (Jean $6,12)$. Ces morceaux nous rappellent que la fécondation est toujours réciproque, mutuelle. La rencontre avec l'autre est la nourriture qui fait grandir la graine des plantes, et ces plantes vont étendre leurs racines et vont les nouer avec les autres racines dans la terre. Ce contact, cette relation n'est pas le patrimoine d'une seule tradition, mais il est le fond invisible qui contient et entoure toute singularité, en la gardant dans son apparaître et son disparaître.

\section{Références}

Brunner, F. (1969), Maître Eckhart, Paris, Seghers.

De Cues, N. (1986) [2007, 2009], Le Tableau ou la vision de Dieu, Paris, Cerf.

Derrida, J. (1996), Le monolinguisme de l'autre, Paris, Galilée.

Ghilardi, M. (2005), "Eterotopie culturali in Nishida Kitarō e Mou Zongsan ", Simplègadi. Rivista di filosofia interculturale, 10/26, p. 131168.

(2009), Una logica del vedere. Estetica ed etica nel pensiero di Nishida Kitarō, Milan-Udine, Mimesis.

(2012), «Postfazione: L'infinito ritrarsi dello sfondo ", dans K. NishidA, Luogo / trad. par E. Fongaro, Milan-Udine, Mimesis, p. 135145. 
Heisig, J. W. (2000), «Non-I and Thou: Nishida, Buber, and the Moral Consequences of Self-actualization ", Philosophy East and West, 50/2, p. 179-207.

- (2001), Philosophers of Nothingness, Honolulu, University of Hawaii Press.

Jullien, F. (2008), De l'universel, de l'uniforme, du commun et du dialogue entre les cultures, Paris, Fayard.

Lossky, V. (1973), Théologie négative et connaissance de Dieu chez Maître Eckhart, Paris, Vrin.

Maître Eckhart (I97I), Sermons, vol. I, introduction et traduction de Jeanne Ancelet-Hustache, Paris, Seuil.

(1963), «Deutsche Werke», Bd. V, Die deutschen und lateinischen Werke, hrsg. Josef Quint, Stuttgart, Kohlhammer.

Merleau-Ponty, M. (1964a), L'œil et l'esprit, Paris, Folio/Essais. (1964b), Le visible et l'invisible, Paris, Gallimard.

Nishida, K. (1911), Zen no kenkyū 善の研究, dans Nishida Kitarō Zenshū 西田幾多郎全集, vol. I, Tōkyō, Iwanami, 1965-1988, p. 1-200.

Nishida, K. (1923), Geijutsu to dōtoku 芸術と道徳, dans Nishida Kitarō Zenshū, vol. III, Tōkyō, Iwanami, 1965-1988, p. 239-545.

Nishida, K. (1926), «Basho» 場所, dans Nishida Kitarō Zenshū, vol. IV, Tōkyō, Iwanami, 1965-1988, p. 208-289 (pour les citations nous avons suivi le vol. III, Tōkyō, Iwanami, 2002-2009, p. 415-477).

Nishida, K. (2003), L’Éveil à soi, trad., introduction et notes par J. Tremblay, Paris, CNRS.

Panikkar, R. (2002), "Soleil levant et Soleil couchant », Diogène, 200, p. 5-16. Book.

(2008), «Mito, simbolo, culto », Opera omnia, IX, 1, Milan, Jaca (2009), «Pluralismo e interculturalità », Opera omnia, VI, 1, Milan, Jaca Book.

SINI, C. (1985), Immagini di verità, Milan, Spirali.

Suzuki, D.T. (1957), Mysticism: Christian and Buddhist. The Eastern and Western Way, New York, Macmillan.

Tremblay, J. (2000), Nishida Kitarō. Le jeu de l'individuel et de l'universel, Paris, CNRS. 
(2003), «Introduction », dans K. NishidA, L'éveil à soi, Paris, CNRS, p. 9-51.

(2007a), Introduction à la philosophie de Nishida, Paris, L'Harmattan.

(2007b), L'être soi et l'être-ensemble. L'autoéveil comme méthode philosophique chez Nishida, Paris, L'Harmattan.

UEDA, S. (1965), Die Gottesgeburt in der Seele und der Durchbruch zur Gottheit. Die mystische Anthropologie Meister Eckharts und ibre Konfrontation mit der Mystik des Zen-Buddhismus, Gütersloh, Mohn.

UedA, S. (1977), "Das “Nichts” bei Meister Eckhart und im Zen Buddhismus unter besondere Berucksichtigung des Grenzbereiches von Theologie und Philosophie », dans D. Papenfuss et J. Söring., dir., Transzendenz und Immanenz. Philosophie und Theologie in der veranderten Welt, Stuttgart, Kohlhammer, p. 257-266.

UEDA, S. (1989), "Eckhart und Zen am problem 'Freiheit und Sprache' », dans M. KRAATZ, dir., Luther und Shinran - Eckhart und Zen. Joachim Wach - Vorlesungen der Philipps-Universität Marburg, Köln, E.J. Brill, p. 21-92.

Vannini, M. (2011), Dialettica della fede, Firenze, Le Lettere.

WACKernagel, W. (1991), Ymagine denudari. Éthique de l'image et métaphysique de l'abstraction chez Maître Eckhart, Paris, Vrin.

(1998), "L'être des images », dans E. Zum Brunn, Voici Maître Eckhart, Grenoble, Jérôme Millon, p. 455-472.

\section{Résumé}

Cet article porte sur l'affinité de pensée entre le maître dominicain Johannes Eckhart (1260-1327) et le philosophe japonais Nishida Kitarō (1870-1945) à propos de la dimension de l'image par rapport au questionnement de ce qui se trouve au-delà de la parole, mais qui demeure comme l'objet central de la recherche théologique et philosophique pour les deux penseurs. L'attitude qui soutient une rencontre entre les pensées de Eckhart et Nishida peut réduire les prétentions absolutistes et identitaires qui empêchent un renouvellement de la philosophie et de la théologie. La vérité qui apparaît dans la rencontre de deux penseurs est une vérité qui reste une, mais pluraliste, puisqu'elle sait respecter la différence des identifications religieuses et philosophiques - il ne s'agit pas de les nier en mêlant tout dans un même système indifférencié - et à la fois elle sait garder un fond qui résiste audelà ou en deçà des différences. 


\section{Abstract}

This essay focuses on some affinities between the thoughts of the Dominican theologian Johannes Eckhart (1260-1327) and the Japanese philosopher Nishida Kitarō (1870-1945). In particular, the theme of «image» is considered as a central issue to create some links and interrelations: for both thinkers we can't avoid the dialectics between what lies beyond thought and the inner limits means of the practice of thinking, when confronted to its highest goal - the Absolute, or God. The attitude we can witness in studying and crossing the two experiences of thought can enhance the efforts for an intercultural renewal of philosophy and theology. The truth that appears remains one, but pluralist: it can respect the difference of the religious and philosophical identifications, without claiming to bring every single specificity in one undifferentiated system, and at the same time it can take care for what lies beyond every difference. 